PROCEEDINGS OF THE

AMERICAN MATHEMATICAL SOCIETY

Volume 124, Number 12, December 1996, Pages 3851-3856

S 0002-9939(96)03681-7

\title{
STABLE PERIODIC PROJECTIVE PLANES
}

\author{
MANUEL RITORÉ
}

(Communicated by Peter Li)

\begin{abstract}
We find all stable projective planes with finite topology which are properly embedded in $\mathbb{R}^{3} / \Gamma$, where $\Gamma$ is a discrete subgroup of translations in $\mathbb{R}^{3}$. Here stable means second order minimum of the area. The surfaces we obtain are a quotient of the helicoid and quotients of the doubly periodic Scherk surfaces.
\end{abstract}

\section{INTRODUCTION}

Minimal surfaces are the critical points of the area for any variation with compact support. A minimal surface is said to be stable if the second derivative of the area is nonnegative for the above type of variations. The purpose of this paper is to study stable minimal surfaces properly embedded in quotients of $\mathbb{R}^{3}$ by discrete subgroups $\Gamma$ of translations.

We know from the results of Fischer-Colbrie and Schoen [3] and Fischer-Colbrie [2] that a complete orientable stable surface immersed in a complete orientable flat 3 -manifold must be totally geodesic. However many examples of stable nonorientable surfaces embedded in flat three-tori are known, see the paper by M. Ross $[13$, p. 73$]$. We treat the nonorientable case and we obtain in Theorem 3.2 the following result:

The only stable projective planes with finite topology which are properly embedded in $\mathbb{R}^{3} / \Gamma$, where $\Gamma$ is a discrete subgroup of translations in $\mathbb{R}^{3}$, are

(i) a one-ended surface with total curvature $-2 \pi$ obtained as a quotient of the helicoid, or

(ii) two-ended surfaces with total curvature $-2 \pi$ obtained as quotients of doubly periodic Scherk surfaces.

Recall that a surface has finite topology if it is homeomorphic to a compact surface with a finite number of points removed. We also obtain some restrictions on the number and type of ends of a noncompact stable surface with finite topology properly embedded in $\mathbb{R}^{3} / \Gamma$, with $\Gamma$ a discrete subgroup of translations. We prove in Proposition 3.1 that

Received by the editors June 12, 1995.

1991 Mathematics Subject Classification. Primary 53A10; Secondary 49Q20.

Key words and phrases. Minimal surfaces, stable surfaces.

Research partially supported by DGICYT grant PB94-0796.

(C)1996 American Mathematical Society 
(i) if $\operatorname{rank}(\Gamma)=2$ then the surface has two ends of Scherk type, and

(ii) if $\operatorname{rank}(\Gamma)=1$ then the surface has either one planar type end or one helicoidal type end with winding number 1.

We recall that M. Ross has proved [13, Theorem 3] that a complete, regular, nonorientable minimally immersed surface in $\mathbb{R}^{3}$ with finite total curvature cannot be stable. He also obtains $[13$, p. 73$]$ examples of branched stable nonorientable immersions whose orientation covering can be compactified by a hyperelliptic Riemann surface of genus $3+2 n$, with $n \in \mathbb{N}$. If fact when $n=0$ he obtains examples of compact unbranched stable nonorientable surfaces embedded in flat three-tori. These surfaces were studied by W.H. Meeks [8, Theorem 7.1]. We should also mention that Choe [1, Theorem 5] proved that any complete nonorientable (branched) minimal surface in $\mathbb{R}^{3}$ with total curvature $-2 \pi$ is stable. In particular, Hennenberg surface is a branched stable projective plane in $\mathbb{R}^{3}$.

In the proof of our results we make use of M. Ross' paper [13] about stable nonorientable surfaces, which is based on ideas by López and Ros [6], and also of the fundamental works by Meeks and Rosenberg on singly [10] and doubly [9] periodic properly embedded minimal surfaces.

We have organized the paper in two sections. In the next one we state some basic facts about stable nonorientable surfaces and properly embedded minimal surfaces and we show the stability of some projective planes. In the last one we prove the results stated in this introduction.

\section{Preliminaries And examples of Stable surfaces}

Suppose that $M$ is a nonorientable minimal surface immersed in $\mathbb{R}^{3} / \Gamma$ and that $M^{\prime} \rightarrow M$ is the orientation covering. Call $I: M^{\prime} \rightarrow M^{\prime}$ the interchange of sheets. Then $M^{\prime}$ is immersed in $\mathbb{R}^{3} / \Gamma$ and we have that $M$ is stable if and only if

$$
\int_{M^{\prime}}-u(\Delta u-2 K u) \geq 0
$$

for all smooth $u: M^{\prime} \rightarrow \mathbb{R}$ with compact support on $M^{\prime}$ and $u \circ I=-u$. Here $\Delta$ is the Laplacian and $K$ is the Gauss curvature on $M^{\prime}$. See [13] and the references cited there.

Now we briefly recall some basic facts about properly embedded minimal surfaces.

Let $M \subset \mathbb{R}^{3} / \Gamma$ be a properly embedded nonorientable minimal surface. The surface $M$ is not totally geodesic, since otherwise it would be a quotient of a plane in $\mathbb{R}^{3}$ by $\Gamma$ and so it would be orientable.

Let $\bar{M}$ be the pullback of $M$ to $\mathbb{R}^{3}$ via the map $\mathbb{R}^{3} \rightarrow \mathbb{R}^{3} / \Gamma$. The surface $\bar{M}$ is properly embedded and, by the strong halfspace theorem of Hoffman and Meeks [4], it is connected. Consider the subgroup $\Gamma^{\prime} \subset \Gamma$ of those translations in $\Gamma$ that are orientation preserving when restricted to $\bar{M}$. Then $M^{\prime}=\bar{M} / \Gamma^{\prime}$ is an orientable properly embedded minimal surface in $\mathbb{R}^{3} / \Gamma^{\prime}$ and there are induced twofold coverings $\mathbb{R}^{3} / \Gamma^{\prime} \rightarrow \mathbb{R}^{3} / \Gamma, M^{\prime} \rightarrow M$. The last one is equivalent to the orientation covering of $M$. The surface $M^{\prime}$ is invariant by an order two translation $T$ in $\mathbb{R}^{3} / \Gamma^{\prime}$, and $M=M^{\prime} / T$. The sheet interchange $I$ is the restriction of $T$ to $M^{\prime}$. If $(g, \omega)$ is the Weierstrass representation of $M^{\prime}$ then we have that $g \circ I=-1 / \bar{g}$ and $\overline{I^{*} \omega}=-g^{2} \omega$, see $\left[7\right.$, Section 2]. We have also that $T$ and $\Gamma^{\prime}$ generate $\Gamma$. 
Reciprocally if $M^{\prime} \subset \mathbb{R}^{3} / \Gamma^{\prime}$ is an orientable properly embedded minimal surface with Weierstrass representation $(g, \omega)$, see [7], and $I: M^{\prime} \rightarrow M^{\prime}$ is an anticonformal involution on $M^{\prime}$ without fixed points and such that

$$
\begin{aligned}
g \circ I & =-1 / \bar{g}, \\
\overline{I^{*} \omega} & =-g^{2} \omega,
\end{aligned}
$$

then $I$ extends to a nontrivial translation $T$ on $\mathbb{R}^{3} / \Gamma^{\prime}$ such that $T \circ T$ is the identity on $\mathbb{R}^{3} / \Gamma^{\prime}$; see the arguments in $\left[7\right.$, p. 527]. The group generated by $T$ and $\Gamma^{\prime}$ is a discrete subgroup $\Gamma$ with the same rank as $\Gamma^{\prime}$, and the quotient $M^{\prime} / T$ is a properly embedded nonorientable minimal surface in $\mathbb{R}^{3} / \Gamma$.

We shall apply the above discussion to some concrete surfaces.

Consider the helicoid $M^{\prime}$ that is conformally parameterized by $\mathbb{C}^{*}$ with Weierstrass representation

$$
g=z, \quad \omega=\frac{i d z}{z^{2}} .
$$

This is a minimal surface properly embedded in $\mathbb{R}^{3} / \Gamma^{\prime}$, where $\Gamma^{\prime}$ is the group generated by a nontrivial vector. The anticonformal involution $I(z)=-1 / \bar{z}$ has no fixed points, and (2) holds. So $I$ extends to a translation $T$ and $M^{\prime} / T$ is a nonorientable helicoid $\mathcal{H}$ with total curvature $-2 \pi$ and one end which is properly embedded in some $\mathbb{R}^{3} / \Gamma$, where $\operatorname{rank}(\Gamma)=1$.

Now consider the Scherk surfaces whose Weierstrass representation is given on $M^{\prime}=\overline{\mathbb{C}}-\left\{ \pm e^{ \pm i \theta}\right\}$ by

$$
g=z, \quad \omega=\frac{i d z}{\left(z-e^{i \theta}\right)\left(z-e^{-i \theta}\right)\left(z+e^{i \theta}\right)\left(z+e^{-i \theta}\right)} .
$$

These surfaces are embedded in $\mathbb{R}^{3} / \Gamma^{\prime}$, where $\Gamma^{\prime}$ (depending on $\theta$ ) is a rank-two subgroup of translations in $\mathbb{R}^{3}$. The anticonformal involution $I(z)=-1 / \bar{z}$ has no fixed points, and we have that (2) holds. So we conclude that $I$ extends to an order two translation $T$ on $\mathbb{R}^{3} / \Gamma$ and that $M^{\prime} / T$ is a nonorientable minimal surface embedded in $\mathbb{R}^{3} / \Gamma$, where $\Gamma$ is the rank two subgroup generated by $T$ and $\Gamma^{\prime}$. We shall refer to these surfaces as Scherk projective planes.

Note that the condition $g \circ I=-1 / \bar{g}$ implies that $I$ is the only anticonformal involution on the helicoid and on the Scherk surfaces that gives a nonorientable quotient.

The following result will be used to prove that these surfaces are stable.

Proposition 2.1. Let $M$ be a nonorientable minimal surface in a flat 3-manifold and let $M^{\prime}$ be the orientation covering. If $M^{\prime}$ has index one then $M$ is stable.

Proof. Since $M$ is nonorientable we have to prove that (1) holds for smooth $u$ on $M^{\prime}$ with compact support and $u \circ I=-u$. The surface $M^{\prime}$ has index one if for any exhaustion $\mathcal{U}$ of $M^{\prime}$ by bounded domains we have that

$$
\sup \{\operatorname{index}(L, \Omega) ; \Omega \in \mathcal{U}\}=1 \text {, }
$$

where index $(L, \Omega)$ is the number of negative eigenvalues of the self-adjoint elliptic operator $L=\Delta-2 K$ on $\Omega$, see [2]. Consider an exhaustion $\mathcal{U}$ of $M^{\prime}$ by $I$ invariant domains. Let $u$ be smooth with compact support and $u \circ I=-u$. Choose $\Omega \in \mathcal{U}$ with $\operatorname{supp}(u) \subset \Omega$. Then $\operatorname{index}(L, \Omega)=0,1$. If the index is 0 then the variational characterization of the first eigenvalue shows that (1) holds for $u$. If $\operatorname{index}(L, \Omega)=1$ then the first eigenfunction of the operator $L$ in $\Omega$ is invariant by $I$ 
and so is $L_{2}$-orthogonal to $u$. The variational characterization of the non-negative second eigenvalue of $L$ on $\Omega$ then shows that (1) holds for $u$.

Corollary 2.2. The nonorientable helicoid $\mathcal{H}$ and Scherk projective planes are stable.

Proof. It follows from the results of Montiel and Ros [11, Corollary 22] that the orientation coverings have index one, and we conclude by applying the above proposition.

Remark. When the total curvature of $M^{\prime}$ is $-4 \pi$, Choe [1, Lemma 2] has proved, using results of Barbosa and do Carmo, that $M$ is stable.

\section{Main Results}

Proposition 3.1. Let $M$ be a stable nonorientable minimal surface properly embedded in $\mathbb{R}^{3} / \Gamma$ with finite total curvature. Suppose that $M$ is not compact.

Then $M$ has at most two ends. Moreover

(i) if $\operatorname{rank}(\Gamma)=2$ then $M$ has two ends, which are of Scherk type;

(ii) if $\operatorname{rank}(\Gamma)=1$ then $M$ has either one planar type end or one helicoidal type end whose winding number equals 1.

Remark. When $\operatorname{rank}(\Gamma)=2$ what we call Scherk type ends were called ends asymptotic to flat vertical annuli in the paper of Meeks and Rosenberg [9]. These are the only possible type of ends for properly embedded doubly periodic minimal surfaces.

See [10, Proposition 4.1] for the definition of Scherk, helicoidal and planar type ends when the rank of $\Gamma$ is one, and [10, p. 539] for a discussion of the winding numbers of these ends.

Proof. Since there are no nonorientable minimal surfaces properly embedded in $\mathbb{R}^{3}$ we have that $\Gamma \neq\{0\}$. We also have that $\operatorname{rank}(\Gamma) \neq 3$ as $M$ is properly embedded and noncompact. The surface $M$ is not flat, since otherwise it would be a quotient of a plane in $\mathbb{R}^{3}$ by $\Gamma$ and so it would be orientable.

Consider the orientation covering $M^{\prime}$ of $M$ and the Gauss map $\phi: M^{\prime} \rightarrow \mathbb{S}^{2}$. The surface $M^{\prime}$ is properly embedded in $\mathbb{R}^{3} / \Gamma^{\prime}$ with finite total curvature. By $[9$, Proposition 1] and [10, Theorem 1] we have that $M^{\prime}$ is conformally equivalent to a finitely punctured compact Riemann surface $\bar{M}$ of genus $\gamma$. The Gauss map $\phi$ extends to $\bar{M}$ by [9, Proposition 1] and [10, p. 540].

By [13, Theorem 1] we obtain that deg $\phi \leq \gamma+1$, and hence the total curvature $C\left(M^{\prime}\right)=\int_{M^{\prime}} K$ of $M^{\prime}$ satisfies

$$
C\left(M^{\prime}\right)=-4 \pi \operatorname{deg} \phi \geq-4 \pi(\gamma+1) .
$$

Let $\left\{p_{1}, \ldots, p_{s}, I\left(p_{1}\right), \ldots, I\left(p_{s}\right)\right\}$ be the ends of $M^{\prime}$. The results of Meeks and Rosenberg [9, Theorem 2], [10, Theorem 4] imply that

$$
C\left(M^{\prime}\right)=2 \pi\left(\chi\left(M^{\prime}\right)-W\left(M^{\prime}\right)\right),
$$

where the integer number $W\left(M^{\prime}\right) \geq 0$ is the total winding number of $M^{\prime}$, defined as the sum of the winding numbers at the ends of $M^{\prime}$, see [10, p. 539]. We take $W\left(M^{\prime}\right)=0$ if $\operatorname{rank}(\Gamma)=2$. Note that $W\left(M^{\prime}\right)$ is even since the ends at $p_{i}, I\left(p_{i}\right)$ are of the same type. Since $\chi\left(M^{\prime}\right)=2-2 \gamma-2 s$ we have from (3) and (4) that

$$
0 \leq s+W\left(M^{\prime}\right) / 2 \leq 2 .
$$


Let us prove (i). Suppose that $\operatorname{rank}(\Gamma)=2$. Recall that in this case $W\left(M^{\prime}\right)=0$. We have that $M^{\prime}$ is an orientable minimal surface properly embedded with $2 s$ ends and, by [9, Theorem 3], $2 s \geq 4$. Hence $s=2$ and $M^{\prime}$ has two ends.

Now we prove (ii). Suppose that $\operatorname{rank}(\Gamma)=1$. By (5) we have that $s=1,2$ since $M^{\prime}$ is not compact. Consider first the case $s=2$. Then $M^{\prime}$ has four Scherk type ends and the translation $T$ of order two in $\mathbb{R}^{3} / \Gamma$ leaves these ends invariant. This is a contradiction since $M=M^{\prime} / T$ and $T$ restricted to $M^{\prime}$ coincides with $I$, which has no fixed ends. Hence the case $s=2$ is not possible.

If $s=1$ we have from (5) that $W\left(M^{\prime}\right)=0,2$. If $W\left(M^{\prime}\right)=0$ then $M^{\prime}$ has two Scherk type ends, and lifting the surface to $\mathbb{R}^{3}$ we get a nonflat properly embedded minimal surface contained in a halfspace. This contradicts the halfspace theorem [4] and proves that the case $W\left(M^{\prime}\right)=0$ is not possible. Suppose that $W\left(M^{\prime}\right)=2$. Since all the ends of $M^{\prime}$ are of the same type we have that $M$ has either one planar type end or one helicoidal type end with winding number equal to 1 by [10, p. 539], which proves (ii).

Remark. The above discussion shows that only the cases $s=2, W\left(M^{\prime}\right)=0$ and $s=1, W\left(M^{\prime}\right)=1$ are possible. Hence equality holds in (5) and so the total curvature of $M$ is $-2 \pi(\gamma+1)$ (that of $M^{\prime}$ is $-4 \pi(\gamma+1)$ ).

Theorem 3.2. Let $M \subset \mathbb{R}^{3} / \Gamma$ be a stable properly embedded projective plane with finite topology. Then up to scaling and rigid motion we have that either

(i) $M$ is a Scherk projective plane, or

(ii) $M$ is the nonorientable helicoid $\mathcal{H}$.

Proof. We observe first that $M$ cannot be compact, since there are no minimally immersed projective planes in quotients of $\mathbb{R}^{3}$.

Suppose first that $M$ has finite total curvature. By Proposition 3.1 we have that $M$ has at most two ends.

If $M$ has two ends then $M^{\prime}$ is a genus zero minimal surface with four ends properly embedded in $\mathbb{R}^{3} / \Gamma$, with $\operatorname{rank}(\Gamma)=2$ by Proposition 3.1. These ends cannot be parallel, since if they were the map between the fundamental groups $\pi_{1}\left(M^{\prime}\right) \rightarrow \pi_{1}\left(\mathbb{R}^{3} / \Gamma\right)$ would be nonsurjective [9, Theorem 3], providing then a contradiction again by the halfspace theorem. So the ends are not parallel, and then the arguments in $\left[9\right.$, p. 369] imply that $M^{\prime}$ is, up to scaling and rigid motion, one of the doubly periodic Scherk surfaces whose Weierstrass representation is the one given in the previous section. So $M$ is a Scherk projective plane which is stable by Corollary 2.2.

If $M$ has only one end we have that $W\left(M^{\prime}\right)=2$, that $M^{\prime}$ has two ends which are either of planar or helicoidal type, and that the surface $M^{\prime}$ is properly embedded in $\mathbb{R}^{3} / \Gamma^{\prime}$, where $\operatorname{rank}\left(\Gamma^{\prime}\right)=1$. If $M^{\prime}$ has two planar type ends then the lifting of $M^{\prime}$ to $\mathbb{R}^{3}$ would be a properly embedded minimal surface in $\mathbb{R}^{3}$ contained in a halfspace, a contradiction as before. If $M^{\prime}$ has two helicoidal type ends then it must be a helicoid by Toubiana's theorem [14], see also [12, Theorem 5]. Proposition 3.1 shows that the winding number at the ends equals one and so the Weierstrass representation of this helicoid, up to scaling and rigid motion, is given by $g=z, \omega=i d z / z^{2}$. This implies that $M$ is, up to scaling and rigid motion, the nonorientable helicoid $\mathcal{H}$.

If $M$ has infinite total curvature then it follows from the work of Lima and Silveira [5] that $M$ is unstable.

Remark. We do not know if these stable surfaces are area-minimizing. 
Remark. From the results of M. Ross [13, Corollary 6] and Lima and Silveira [5] it can be deduced that there are neither stable properly embedded Klein bottles nor stable surfaces with Euler characteristic -1 which are finitely punctured.

Added, June 1996. We have recently learnt that Marty Ross and Chad Schoen, Stable quotients of periodic minimal surfaces, Communications in Analysis and Geometry, have proved stronger results than ours.

\section{REFERENCES}

[1] J. Choe, Index, vision number and stability of complete minimal surfaces, Arch. Rat. Mech. Anal. 109, no. 3 (1990) 195-212. MR 91b:53007

[2] D. Fischer-Colbrie, On complete minimal surfaces with finite Morse index in threemanifolds, Invent. Math. 82 (1985) 121-132. MR 87b:53090

[3] D. Fischer-Colbrie, R. Schoen, The structure of complete stable minimal surfaces in 3manifolds of nonnegative scalar curvature, Comm. Pure Applied Math. 33 (1980) 199-211. MR 81i: 53044

[4] D. Hoffman, W.H. Meeks, The strong halfspace theorem for minimal surfaces, Invent. Math. 101 (1990) 373-377. MR 92e:53010

[5] I.C. Lima, A.M. da Silveira, Stability of complete nonorientable minimal surfaces in $\mathbb{R}^{3}$, preprint.

[6] F.J. López, A. Ros, Complete minimal surfaces with index one and stable constant mean curvature surfaces, Comment. Math. Helv. 64 (1989) 34-43. MR 90b:53006

[7] W.H. Meeks, The classification of complete minimal surfaces in $\mathbb{R}^{3}$ with total curvature greater than $-8 \pi$, Duke Math. J. 48 (1981) 523-535. MR 82k:53009

[8] W.H. Meeks, The theory of triply periodic minimal surfaces, Indiana U. Math. J. 39 (1990) 877-936. MR 92e:53012

[9] W.H. Meeks, H. Rosenberg, The global theory of doubly periodic minimal surfaces, Invent. Math. 97 (1989) 351-379. MR 90m:53017

[10] W.H. Meeks, H. Rosenberg, The geometry of periodic minimal surfaces, Comment. Math. Helv. 68 (1993) 538-578. MR 95a:53011

[11] S. Montiel, A. Ros, Schrödinger operators associated to a holomorphic map, Proceedings Conference on Global Differential Geometry and Global Analysis, Lecture Notes on Mathematics 1481 (1991) 147-174. MR 93k:58053

[12] J. Pérez, A. Ros, Some uniqueness and nonexistence results for embedded minimal surfaces, Math. Ann. 295 (1993) 513-525. MR 94a:53020

[13] M. Ross, Complete nonorientable minimal surfaces in $\mathbb{R}^{3}$, Comment. Math. Helvetici 67 (1992) 64-76. MR 92k:53022

[14] E. Toubiana, Un théorème d'unicité de l'helicoïde, Annales de L'Institut Fourier 38 (1988) 121-132. MR 90a:53015

Departamento de Geometría y Topología, Universidad de Granada, E-18071, Granada, SPAIN

E-mail address: ritore@goliat.ugr.es 